\title{
Gelatinous pseudocysts in cryptococcal infection of the central nervous system
}

\author{
Joy Zhuo Ding MD, Carlos Torres MD
}

Cite as: CMAJ 2021 April 12;193:E522. doi: 10.1503/cmaj.201547

A 44-year-old man from British Columbia was admitted to hospital with a 2-week history of increasing headaches, vomiting, and substantial neck and back pain. The patient was immunosuppressed because he was taking ocrelizumab, azathioprine and prednisone for multiple sclerosis (MS) and immune thrombocytopenic purpura. He had no nuchal rigidity or fever, and his clinical examination was unchanged from his baseline of stable neurological deficits related to his MS. Magnetic resonance imaging (MRI) of his brain with gadolinium showed tiny cysts in the basal ganglia, which were not present in an MRI performed 4 weeks earlier (Figure 1A; Appendix 1, Supplemental Figure, available at www.cmaj.ca/ lookup/doi/10.1503/cmaj.201547/tab-related-content).

We delayed a lumbar puncture because of anticoagulation treatment. A biopsy of an enlarging mass in the right lower lobe of the lung identified Cryptococcus gattii. A lumbar puncture later showed normal cerebrospinal fluid (CSF) results (leucocyte count of 2 [normal range $0-5$ ] $\times 10^{6} / \mathrm{L}$, red blood cell count of 4 [normal range $<1$ ] $\times 10^{6} / \mathrm{L}$, glucose level of 3.6 [normal range 2.2-3.9] $\mathrm{mmol} / \mathrm{L}$ and protein level of 0.48 [normal range 0.12-0.6] g/L), but opening pressure exceeded $55 \mathrm{~cm} \mathrm{H}_{2} \mathrm{O}$. The CSF was positive for cryptococcal antigen. Despite appropriate treatment with amphotericin B and flucytosine, as well as daily lumbar punctures, a brain MRI 2 weeks after admission showed a substantial increase in size and number of gelatinous pseudocysts (Figure 1B).

Four months later, we discharged the patient from hospital and continued his fluconazole treatment; however, he had persistent profound visual loss.

Cryptococcus gattii, a fungus found in trees and soil, is endemic to BC and infects humans through spore inhalation. Vancouver Island has one of the highest annual incidence rates, varying between 0.1 and 2.2 per 100000 population in 2003-2018. ${ }^{1}$ Older age is a risk factor for infection and patients who are immunocompetent are frequently infected.

Findings using MRI in cryptococcal meningoencephalitis include parenchymal cryptococcomas, gelatinous pseudocysts, leptomeningeal enhancement and communicating hydrocephalus. ${ }^{2}$ Gelatinous pseudocysts are found in almost $50 \%$ of HIV-associated C. gattii infections; however, its prevalence in immunocompetent patients is unknown. ${ }^{3}$ Gelatinous pseudocysts form over weeks but may be difficult to recognize initially, especially in patients with pre-existing MRI abnormalities.

Physicians should consider CNS C. gattii infection in both immunocompetent and immunocompromised patients with headaches, neck pain and lung lesions who have visited Vancouver Island. Appearance of gelatinous pseudocysts or other typical MRI findings are helpful if present. Composition of the CSF may be normal; cryptococcal antigen testing and cultures of the CSF are required to confirm the diagnosis.

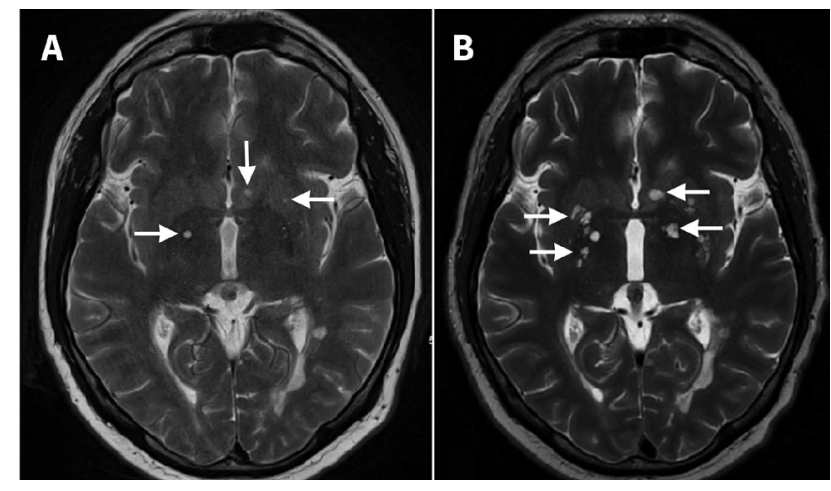

Figure 1: Consecutive axial $T_{2}$-weighted images of the brain at the level of the third ventricle in a 44-year-old man with cryptococcal infection showing (A) interval development of 3 tiny pseudocysts (arrows) in a follow-up MRI performed 4 weeks later ( 2 wk after symptom onset) and (B) substantial interval increase in the size and number of the gelatinous pseudocysts in the bilateral basal ganglia (arrows), in a follow-up MRI obtained 2 weeks later (4 wk after symptom onset).

\section{References}

1. Reportable Diseases Data Dashboard. Vancouver: BC Centre for Disease Control. Available: www.bccdc.ca/health-professionals/data-reports/reportable-diseases -data-dashboard?Disease=Cryptococcus\%20gattii (accessed 2020 Sept. 20).

2. Duarte SB, Oshima MM, do Amaral Mesquita JV, et al. Magnetic resonance imaging findings in central nervous system cryptococcosis: comparison between immunocompetent and immunocompromised patients. Radiol Bras 2017;50:359-65.

3. Chen SC-A, Meyer W, Sorrell TC. Cryptococcus gattii infections. Clin Microbiol Rev 2014;27:980-1024.

Competing interests: None declared.

This article has been peer reviewed.

The authors have obtained patient consent.

Affiliations: Division of Neurology (Ding), Penticton Regional Hospital, Penticton, BC; Department of Radiology (Torres), University of Ottawa; Department of Medical Imaging (Torres), The Ottawa Hospital, Ottawa, Ont.

Content licence: This is an Open Access article distributed in accordance with the terms of the Creative Commons Attribution (CC BY-NC-ND 4.0) licence, which permits use, distribution and reproduction in any medium, provided that the original publication is properly cited, the use is noncommercial (i.e., research or educational use), and no modifications or adaptations are made. See: https://creativecommons.org/licenses/by-nc-nd/4.0/

Correspondence to: Joy Ding, joy.ding@medportal.ca 